\title{
Hopf-bifurcations on a Nonlinear Chaotic Discrete Model
}

\author{
Nabajyoti Das \\ Dept. of Mathematics \\ Jawaharlal Nehru College, Boko \\ Kamrup, India
}

\author{
Tarini Kumar Dutta \\ Department of Mathematics \\ Gauhati University, Guwahati \\ Guwahati, India
}

\begin{abstract}
In this paper we highlight some analytical and numerical discussion of Hopf bifurcation for the nonlinear two-dimensional chaotic map in the plane $f: \mathfrak{R}^{2} \rightarrow \mathfrak{R}^{2}$ given by $f(x, y)=\left(a-b x+x^{2} y, b x+y-x^{2} y\right)$ where the adjustable parameters $a, b \in \mathfrak{R}$. Here we firstly show that if the nonlinear map $f$ undergoes supercritical Hopf bifurcation, then $f^{2}$ undergoes subcritical Hopf bifurcation. Secondly, we show that our numerical and graphical investigations have established some fascinating observation between Hopf bifurcation and Period-doubling bifurcation.
\end{abstract}

Key Words: Supercritical Hopf bifurcation / Subcritical Hopf bifurcation / Period-doubling bifurcation / Nonlinear / Chaotic.

2010 AMS Classification: 37 G 15, 37 G 35, 37 C 45

\section{INTRODUCTION}

In case of one-dimensional maps, the lack of hyperbolicity is usually a signal for the occurrence of bifurcations. In these systems, bifurcations occur when the eigenvalues of a periodic point is either +1 (the saddle node bifurcation) or -1 (the period-doubling bifurcation). For higher dimensional systems, these types of bifurcations also occur, but there are other possible bifurcations of periodic points as well. The most typical of these is the Hopf bifurcation. Although this type of bifurcation was known and understood by the great French mathematician Jules Henri Poincaré as well as the Soviet mathematician Aleksandr Andronov, but Eberhadr Hopf (the German mathematician) was the first to extend these ideas to higher dimensional state spaces.

In the theory of bifurcations, a Hopf bifurcation refers to the local birth and death of a periodic solution as a pair of complex conjugate eigenvalues of the linearization around the fixed point which crosses the imaginary axis of the complex plane as the parameter varies. Under reasonably generic assumptions about the dynamical system, we can expect to see a small amplitude limit cycle branching from the fixed point $[1-3,5-8]$.

We now highlight some useful concepts which are absolutely useful for our purpose.

\subsection{DISCRETE DYNAMICAL SYSTEMS}

Any $C^{k}(k \geq 1)$ map $E: U \rightarrow \Re^{\mathrm{n}}$ on the open set $U \subset \mathfrak{R}^{\mathrm{n}}$ defines an $n$-dimensional discrete-time (autonomous) smooth dynamical system by the state equation

$\overline{\mathrm{x}}_{t+1}=E\left(\overline{\mathrm{x}}_{t}\right), t=1,2,3, \ldots .$. where $\overline{\mathrm{x}}_{t} \in \mathfrak{R}^{n}$ is the state of the system at time $t$ and $E$ maps $\overline{\mathrm{x}}_{t}$ to the next state $\overline{\mathrm{x}}_{t+1}$. Starting with an initial data $\overline{\mathrm{x}}_{0}$, repeated applications (iterates) of $E$ generate a discrete set of points (the orbits)

$$
\left\{E^{t}\left(\overline{\mathrm{x}}_{0}\right): t=0,1,2,3, \ldots \ldots\right\} \text {, where } E^{t}(\overline{\mathrm{x}})=\underbrace{E \circ E \circ \ldots \circ E(\overline{\mathrm{x}})}_{t \text { times }}[9] \text {. }
$$

\subsection{Bifurcation}

Bifurcation, as a scientific terminology, has been used to describe significant and qualitative changes that occur in the solution curves of a dynamical system, as the key system parameters are varied. Very frequently, it is used to describe the qualitative stability changes of the solution curves of a nonlinear dynamical system [7]. Many dynamical systems depend on parameters. Normally a gradual variation of a parameter in the system corresponds to the gradual variation of the solutions of the problem. However, there exits a large number of problems for which the number of solutions changes abruptly and the structures of solution manifolds vary dramatically when a parameter passes through some critical values. This kind of phenomenon is called bifurcation and these parameter values are called bifurcation values (or bifurcation points). In the case of a diffeomorphism $E$, period-doubling bifurcations occur when one of the eigenvalues of the derivative $D E^{q}(\overline{\mathrm{x}})=-1$.

Bifurcation theory is a method for studying how solutions of a nonlinear problem and their stability changes as the parameter vary. The onset of chaos is often studied by bifurcation theory. For example, in parameterized families of one-dimensional map, chaos develops via period-doubling cascade [4].

\subsection{The Hopf bifurcation theorem for maps in the plane $\Re^{2}$ :}

Let $E_{b}(\overline{\mathrm{x}} ; b)$, where $b$ is the bifurcation parameter, be a one-parameter family of maps in the plane $\mathfrak{R}^{2}$ satisfying the following conditions: 
(C1) An isolated fixed point $\overline{\mathrm{x}}^{*}(b)$ exists,

(C2) The map $E_{b}$ is $C^{k}(k \geq 3)$ in the neighborhood of $\left(\overline{\mathrm{x}}^{*}\left(b_{0}\right) ; b_{0}\right)$,

(C3) The Jacobian $D_{\overline{\mathrm{x}}} E\left(\overline{\mathrm{x}}^{*}(b) ; b\right)$ matrix possesses a pair of complex, simple

eigenvalues $\eta(b)=\alpha(b)+i \omega(b)$ and $\bar{\eta}(b)$, such that at the critical value $b=b_{0}$

$$
\left|\eta\left(b_{0}\right)\right|=1, \frac{d|\eta(b)|}{d b}\left(b=b_{0}\right)>0
$$

(C4) $\left(\eta\left(b_{0}\right)\right)^{j} \neq 1, j=3,4$.

(Existence) Then there exists a real number $\varepsilon_{0}>0$ and a $C^{k-1}$ function such that

$$
b(\varepsilon)=b_{0}+b_{1} \varepsilon+b_{3} \varepsilon^{3}+O\left(\varepsilon^{4}\right)
$$

such that for each $\varepsilon \in\left(0, \varepsilon_{0}\right]$ the map $E_{b}$ has an invariant manifold $H(b)$, that is,

$$
E(H(b) ; b)=H(b)
$$

The manifold $H(b)$ is $C^{b}$ diffeomorphic to a circle and consists of points at a distance

$$
O\left(|b|^{1 / 2}\right) \text { of } \overline{\mathrm{x}}^{*}(b) \text {, for } b=b(\varepsilon) .
$$

(Uniqueness) Each compact invariant manifold close to $\overline{\mathrm{x}}^{*}(b)$ for $b=b(\varepsilon)$ is contained in $H(b) \bigcup\{0\}$.

(Stability) If $r_{3}<0\left(\right.$ respectively $\left.r_{3}>0\right)$ then for $r<0$ (respectively $r>0$ ), the fixed point $\overline{\mathrm{x}}^{*}(b(\varepsilon))$ is stable (respectively unstable) and for $r>0$ (respectively $r<0$ ), the fixed point $\overline{\mathrm{x}}^{*}(b(\varepsilon))$ is unstable (respectively stable) and the surrounding manifold $H(b(\varepsilon)$ ) is attracting (respectively repelling). When $r_{3}<0$ (respectively $r_{3}>0$ ) the bifurcation at $b=b(\varepsilon)$ is said to be supercritical (respectively subcritical) [10].

Armed with all these ideas and concepts, we now proceed to concentrate to our main aim and objectives.

\section{GENERAL THEORY}

We consider a discrete-time dynamical system $\overline{\mathrm{x}} \mapsto E(\overline{\mathrm{x}} ; b), \overline{\mathrm{x}} \in \mathfrak{R}^{2}$ depending on the parameter $b \in \mathfrak{R}$, where $E$ is smooth. Suppose that for each $b$ near the origin there is a fixed point of $E$, denoted by $\overline{\mathrm{x}}^{*}(b)$, with the Jacobian matrix $D_{\overline{\mathrm{x}}} E\left(\overline{\mathrm{x}}^{*}(b) ; b\right)$ having a single pair of purely imaginary (complex conjugate) eigenvalues, denoted by $\eta(b)$ and $\bar{\eta}(b)$, cross the imaginary axis in the complex eigenplane as the parameter $b$ passes through the origin. The fixed point is then a center for the linearised system, but for the nonlinear system the higher order terms will typically convert it into either an attracting or repelling focus. In the following, we describe a method for determining which of these two alternatives occurs; it involves making a series of coordinate changes to reduce the system to a normal form.

Firstly, by making a linear change of coordinates we can arrange that $\overline{\mathrm{X}}^{*}(b)=(0,0)$ and then in complex notation,

$z=x+i y$ we obtain an expression for the system in the variable $\mathrm{z}$ as

$$
E(z)=\eta z+A_{1} z^{2}+B_{1} z \bar{z}+C_{1} \bar{z}^{2}+M_{1} z^{2} \bar{z}+\ldots
$$

where $A_{1}, B_{1}, C_{1}, M_{1}$ are complex constants.

Secondly, to make a new change of variables to eliminate the quadratic terms, we put $w=z+\mu z^{2}+v z \bar{z}+\rho \bar{z}^{2}$. Then we expand $E(w)$, keeping only terms up to second order (and noting, e.g. that the difference between $z^{2}$ and $w^{2}$ is third order, so $z^{2}$ can be replaced by $w^{2}$, etc.)

$$
\begin{aligned}
E(z) & =E(\mathrm{z})+2 \mu z E(z)+v \bar{z} E(z)+2 \rho \bar{z} E(z \bar{z}) \\
& =\eta z+A_{1} z^{2}+B_{1} z \bar{z}+C_{1} \bar{z}^{2}+2 \mu \eta z^{2}+v \eta \bar{z} \bar{z}+v \bar{\eta} z \bar{z}+2 \rho \bar{\eta}^{2}
\end{aligned}
$$

Again we have

$$
\eta w=\eta z+\mu \eta z^{2}+v \eta z \bar{z}+\rho \eta \bar{z}^{2}
$$

and equating the above expressions for $E(w)$ and $\eta z$ we find that, if we take

$$
\mu=-A_{1} / \eta, v=-B_{1} / \bar{\eta}, \rho=C_{1} /(\eta-2 \bar{\eta}) .
$$

then the system reduces to $E(w)=\eta w+$ terms of $3^{\text {rd }}$ and higher order. Thus we can write

$$
E(w)=\eta w+p w^{3}+q w^{2} \bar{w}+r w \bar{w}^{2}+s \bar{w}^{3}+\ldots
$$

where $p, q, r, s$ are complex numbers.

Thirdly, we eliminate as many of the third order terms as possible (by a similar procedure to that used in second step). We make a change of variable

$$
\tau=w+d w^{3}+e w^{2} \bar{w}+j w \bar{w}^{2}+l^{3}
$$

and choose $d, e, j, l$ so as to eliminate the third order terms. 
Under these situations, the system reduces to the normal form as

$$
E(\tau)=\eta \tau\left(1+H|\tau|^{2}+O\left(|\tau|^{4}\right)\right.
$$

where,

$$
H=\frac{(1-2 \eta) A_{1} B_{1}}{\eta^{2}(\eta-1)}+\frac{B_{1} \bar{B}_{1}}{\eta-1}+\frac{2 C_{1} \bar{C}_{1}}{\eta^{3}-1}+\eta^{-1} M_{1}
$$

Interestingly, Supercritical (respectively Subcritical) Hopf bifurcations occurs when $H<0$ (respectively $H>0$ ). If $H=$ 0 , we need to consider higher order terms to draw the conclusion.

\section{OUR MAIN ANALYTICAL DISCUSSION}

To study the Hopf bifurcation in a concrete way, we now consider a nonlinear two- dimensional map and make the necessary analytic deduction to study their intrinsic properties. Our 2-D map is

$$
f(x, y)=\left(a-b x+x^{2} y, b x+y-x^{2} y\right)
$$

where the parameter $b \in \mathfrak{R}$, and the parameter $a$ is fixed as $a=1$.

Let $\left(x_{0}, y_{0}\right)$ be a fixed point of the map $f$. Let us change to a new coordinate $\left(x^{\prime}, y^{\prime}\right)$ by the relation $x^{\prime}=x-x_{0}, y^{\prime}=y-y_{0}$ so that $(0,0)$ is a fixed point of the map $f$. Now $x=x^{\prime}+x_{0}, y=y^{\prime}+y_{0}$. Then first iteration of the system (1.1) becomes

$$
\begin{aligned}
& \left\{\begin{array}{c}
x_{1}^{\prime}=1-b\left(x^{\prime}+x_{0}\right)+\left(x^{\prime}+x_{0}\right)^{2}\left(y^{\prime}+y_{0}\right)-x_{0} \\
y_{1}^{\prime}=b\left(x^{\prime}+x_{0}\right)+\left(y^{\prime}+y_{0}\right)-\left(x^{\prime}+x_{0}\right)^{2}\left(y^{\prime}+y_{0}\right)-y_{0}
\end{array}\right. \\
& \Rightarrow\left\{\begin{array}{c}
x_{1}^{\prime}=\left(1-x_{0}-b x_{0}+x_{0}^{2} y_{0}\right)+\left(-b+2 x_{0} y_{0}\right) x^{\prime}+x_{0}^{2} y^{\prime} \\
\quad+y_{0} x^{\prime 2}+2 x_{0} x^{\prime} y^{\prime}+x^{\prime 2} y^{\prime} \\
y_{1}^{\prime}=\left(b x_{0}-x_{0}^{2} y_{0}\right)+\left(b-2 x_{0} y_{0}\right) x^{\prime}+\left(1-x_{0}^{2}\right) y^{\prime}+ \\
\quad\left(-y_{0}\right) x^{2}+\left(-2 x_{0}\right) x^{\prime} y^{\prime}+(-1) x^{\prime 2} y^{\prime}
\end{array}\right.
\end{aligned}
$$

For our convenience, we write $x=x^{\prime}, y=y^{\prime}$.

So

$$
\begin{aligned}
& \begin{aligned}
x_{1}^{\prime}= & m_{1}+n_{1} x+p_{1} y+q_{1} x^{2}+r_{1} x y+s_{1} y^{2}+t_{1} x^{3}+u_{1} x^{2} y \\
& +\hat{v}_{1} x y^{2}+w_{1} y^{3}
\end{aligned} \\
& \begin{aligned}
y_{1}^{\prime}= & M_{1}+N_{1} x+P_{1} y+Q_{1} x^{2}+R_{1} x y+S_{1} y^{2}+T_{1} x^{3}+ \\
& U_{1} x^{2} y+V_{1} x y^{2}+W_{1} y^{3}
\end{aligned} \\
& \text { ) }
\end{aligned}
$$

$$
\begin{aligned}
& m_{1}=1-x_{0}-b x_{0}+x_{0}^{2} y_{0}, n_{1}=-b+2 x_{0} y_{0}, p_{1}=x_{0}^{2}, \\
& q_{1}=y_{0}, r_{1}=2 x_{0}, s_{1}=0, t_{1}=0, u_{1}=1, \hat{v}_{1}=0, w_{1}=0, \\
& M_{1}=b x_{0}-x_{0}^{2} y_{0}, \mathrm{~N}_{1}=b-2 x_{0} y_{0}, P_{1}=1-x_{0}^{2}, Q_{1}=-y_{0}, \\
& R_{1}=-2 x_{0}, S_{1}=0, T_{1}=0, U_{1}=-1, V_{1}=0, W_{1}=0,
\end{aligned}
$$

We now iterate the system (1.2). Let $f\left(x_{1}^{\prime}, y_{1}^{\prime}\right)=\left(x_{2}^{\prime}, y_{2}^{\prime}\right)$,

where

$$
\begin{aligned}
& x_{2}^{\prime}=m_{1}+n_{1}\left(m_{1}+n_{1} x+p_{1} y+q_{1} x^{2}+r_{1} x y+s_{1} y^{2}+t_{1} x^{3}+\right. \\
& \left.u_{1} x^{2} y+\hat{v}_{1} x y^{2}+w_{1} y^{3}\right)+p_{1}\left(M_{1}+N_{1} x+P_{1} y+Q_{1} x^{2}+\right. \\
& \left.R_{1} x y+S_{1} y^{2}+T_{1} x^{3}+U_{1} x^{2} y+V_{1} x y^{2}+W_{1} y^{3}\right)+q_{1}\left(m_{1}\right. \\
& \left.+n_{1} x+\ldots\right)^{2}+r_{1}\left(m_{1}+n_{1} x+\ldots\right)\left(M_{1}+N_{1} x+\ldots\right)+s_{1}\left(M_{1}\right. \\
& \left.+N_{1} x+\ldots\right)^{2}+t_{1}\left(m_{1}+n_{1} x+\ldots\right)^{3}+u_{1}\left(m_{1}+n_{1} x+\ldots\right)^{2}\left(M_{1}\right. \\
& \left.+N_{1} x+\ldots\right)+\hat{v}_{1}\left(m_{1}+n_{1} x+\ldots\right)\left(M_{1}+N_{1} x+\ldots\right)^{2}+w_{1}\left(M_{1}+\right. \\
& \left.N_{1} x+\ldots\right)^{3} \\
& y_{2}^{\prime}=M_{1}+N_{1}\left(m_{1}+n_{1} x+p_{1} y+q_{1} x^{2}+n_{1} x y+s_{1} y^{2}+t_{1} x^{3}+\right. \\
& \left.u_{1} x^{2} y+\hat{v}_{1} x y^{2}+w_{1} y^{3}\right)+P_{1}\left(M_{1}+N_{1} x+P_{1} y+Q_{1} x^{2}+R_{1} x y\right. \\
& \left.+S_{1} y^{2}+T_{1} x^{3}+U_{1} x^{2} y+V_{1} x y^{2}+W_{1} y^{3}\right)+Q_{1}\left(m_{1}+n_{1} x+\ldots\right)^{2} \\
& +R_{1}\left(m_{1}+n_{1} x+\ldots\right)\left(M_{1}+N_{1} x+\ldots\right)+S_{1}\left(M_{1}+N_{1} x+\ldots\right)^{2}+ \\
& T_{1}\left(m_{1}+n_{1} x+\ldots\right)^{3}+U_{1}\left(m_{1}+n_{1} x+\ldots\right)^{2}\left(M_{1}+N_{1} x+\ldots\right)+ \\
& V_{1}\left(m_{1}+n_{1} x+\ldots\right)\left(M_{1}+N_{1} x+\ldots\right)^{2}+W_{1}\left(M_{1}+N_{1} x+\ldots\right)^{3}
\end{aligned}
$$

After simplification we find

$$
\begin{aligned}
& x_{2}^{\prime}=\left(m_{1}+m_{1} n_{1}+M_{1} p_{1}+m_{1}^{2} q_{1}+m_{1} M_{1} r_{1}+m_{1}^{2} M_{1} u_{1}\right)+ \\
& \left(n_{1}^{2}+N_{1} p_{1}+2 m_{1} n_{1} q_{1}+M_{1} n_{1} r_{1}+m_{1} N_{1} r_{1}+2 m_{1} M_{1} n_{1} u_{1}+\right. \\
& \left.m_{1}^{2} N_{1} u_{1}\right) x+\left(n_{1} p_{1}+p_{1} P_{1}+2 m_{1} p_{1} q_{1}+M_{1} p_{1} r_{1}+m_{1} P_{1} r_{1}+\right. \\
& \left.2 m_{1} M_{1} p_{1} u_{1}+m_{1}^{2} P_{1} u_{1}\right) y+\left(n_{1} q_{1}+n_{1}^{2} q_{1}+2 m_{1} q_{1}^{2}+p_{1} Q_{1}+\right. \\
& n_{1} N_{1} r_{1}+M_{1} q_{1} r_{1}+m_{1} Q_{1} r_{1}+M_{1} n_{1}^{2} u_{1}+2 m_{1} n_{1} N_{1} u_{1}+ \\
& \left.2 m_{1} M_{1} q_{1} u_{1}+m_{1}^{2} Q_{1} u_{1}\right) x^{2}+\left(2 n_{1} p_{1} q_{1}+n_{1} r_{1}+N_{1} p_{1} \eta_{1}+\right. \\
& n_{1} P_{1} r_{1}+2 m_{1} q_{1} r_{1}+M_{1} r_{1}^{2}+p_{1} R_{1}+m_{1} r_{1} R_{1}+2 M_{1} n_{1} p_{1} u_{1} \\
& \left.+2 m_{1} N_{1} p_{1} u_{1}+2 m_{1} n_{1} P_{1} u_{1}+2 m_{1} M_{1} r_{1} u_{1}+m_{1}^{2} R_{1} u_{1}\right) x y+ \\
& \left(p_{1}^{2} q_{1}+p_{1} P_{1} r_{1}+M_{1} p_{1}^{2} u_{1}+2 m_{1} p_{1} P_{1} u_{1}\right) y^{2}+\left(2 n_{1} q_{1}^{2}+\right. \\
& N_{1} q_{1} r_{1}+n_{1} Q_{1} r_{1}+n_{1}^{2} N_{1} u_{1}+2 M_{1} n_{1} q_{1} u_{1}+2 m_{1} N_{1} q_{1} u_{1}+ \\
& \left.2 m_{1} n_{1} Q_{1} u_{1}\right) x^{3}+\left[1 / 2\left(2 P_{1} q_{1} r_{1}+2 p_{1} Q_{1} r_{1}+2 N_{1} r_{1}^{2}+2 n_{1} r_{1} R_{1}\right.\right. \\
& +2 n_{1} u_{1}+4 n_{1} N_{1} p_{1} u_{1}+P_{1}\left(2 n_{1}^{2}+4 m_{1} q_{1}\right) u_{1}+4 m_{1} p_{1} Q_{1} u_{1}+ \\
& 2 M_{1} r_{1} u_{1}+4 m_{1} N_{1} r_{1} u_{1}+4 m_{1} n_{1} R_{1} u_{1}+q_{1}\left(4 p_{1} q_{1}+4 n_{1} r_{1}+\right. \\
& \left.4 m_{1} u_{1}\right)+M_{1} u_{1}\left(4 p_{1} q_{1}+4 n_{1} r_{1}+4 m_{1} u_{1}\right)+2 p_{1} U_{1}+2 m_{1} r_{1} U_{1} \\
& \left.\left.+2 m_{1}^{2} u_{1} U_{1}\right)\right] x^{2} y+\left[1 / 2\left(4 p_{1} q_{1} r_{1}+2 P_{1} r_{1}^{2}+2 p_{1} r_{1} R_{1}+\right.\right. \\
& 2 N_{1} p_{1}^{2} u_{1}+4 n_{1} p_{1} P_{1} u_{1}+4\left(M_{1} p_{1}+m_{1} P_{1}\right) r_{1} u_{1}+ \\
& \left.\left.4 m_{1} p_{1} R_{1} u_{1}\right)\right] x y^{2}+p_{1}^{2} P_{1} u_{1} y^{3}
\end{aligned}
$$


$y_{2}^{\prime}=\left(M_{1}+m_{1} N_{1}+M_{1} P_{1}+m_{1}^{2} q_{1}+m_{1} M_{1} R_{1}+m_{1}^{2} M_{1} U_{1}\right)$

$+\left(n_{1} N_{1}+N_{1} P_{1}+2 m_{1} n_{1} q_{1}+M_{1} n_{1} R_{1}+m_{1} N_{1} R_{1}+\right.$

$\left.2 m_{1} M_{1} n_{1} U_{1}+m_{1}^{2} N_{1} U_{1}\right) x+\left(N_{1} p_{1}+P_{1}^{2}+2 m_{1} p_{1} q_{1}+\right.$

$\left.M_{1} p_{1} R_{1}+m_{1} P_{1} R_{1}+2 m_{1} M_{1} p_{1} U_{1}+m_{1}^{2} P_{1} U_{1}\right) y+\left(N_{1} q_{1}+\right.$

$n_{1}^{2} q_{1}+2 m_{1} q_{1}^{2}+P_{1} Q_{1}+n_{1} N_{1} R_{1}+M_{1} q_{1} R_{1}+m_{1} Q_{1} R_{1}+$

$\left.M_{1} n_{1}^{2} U_{1}+2 m_{1} n_{1} N_{1} U_{1}+2 m_{1} M_{1} q_{1} U_{1}+m_{1}^{2} Q_{1} U_{1}\right) x^{2}+$

$\left(2 n_{1} p_{1} q_{1}+N_{1} r_{1}+N_{1} p_{1} R_{1}+n_{1} P_{1} R_{1}+2 m_{1} q_{1} r_{1}+M_{1} r_{1} R_{1}\right.$

$+P_{1} R_{1}+m_{1} R_{1}^{2}+2 M_{1} n_{1} p_{1} U_{1}+2 m_{1} N_{1} p_{1} U_{1}+2 m_{1} n_{1} P_{1} U_{1}$

$\left.+2 m_{1} M_{1} r_{1} U_{1}+m_{1}^{2} R_{1} U_{1}\right) x y+\left(p_{1}^{2} q_{1}+p_{1} P_{1} R_{1}+M_{1} p_{1}^{2} U_{1}\right.$

$\left.+2 m_{1} p_{1} P_{1} U_{1}\right) y^{2}+\left(2 n_{1} q_{1}^{2}+N_{1} q_{1} R_{1}+n_{1} Q_{1} R_{1}+n_{1}^{2} N_{1} U_{1}\right.$

$\left.+2 M_{1} n_{1} q_{1} U_{1}+2 m_{1} N_{1} q_{1} U_{1}+2 m_{1} n_{1} Q_{1} U_{1}\right) x^{3}+$

$\left[1 / 2\left(2 P_{1} q_{1} R_{1}+2 p_{1} Q_{1} R_{1}+2 N_{1} r_{1} R_{1}+2 n_{1} R_{1}^{2}+2 N_{1} u_{1}+\right.\right.$

$4 n_{1} N_{1} p_{1} U_{1}+P_{1}\left(2 n_{1}^{2}+4 m_{1} q_{1}\right) U_{1}+4 m_{1} p_{1} Q_{1} U_{1}+2 m_{1} R_{1} U_{1}$

$+4 m_{1} n_{1} R_{1} U_{1}+4 m_{1} N_{1} r_{1} U_{1}+q_{1}\left(4 p_{1} q_{1}+4 n_{1} \eta_{1}+4 m_{1} u_{1}\right)$

$+M_{1}\left(4 p_{1} q_{1}+4 n_{1} r_{1}+4 m_{1} u_{1}\right) U_{1}+2 P_{1} U_{1}+2 M_{1} R_{1} u_{1}+$

$\left.\left.2 m_{1}^{2} U_{1}^{2}\right)\right] x^{2} y+\left[1 / 2\left(4 p_{1} q_{1} r_{1}+2 P_{1} r_{1} R_{1}+2 p_{1} R_{1}^{2}+\right.\right.$

$2 N_{1} p_{1}^{2} U_{1}+4 n_{1} p_{1} P_{1} U_{1}+4\left(M_{1} p_{1}+m_{1} P_{1}\right) r_{1} U_{1}+$

$\left.\left.4 m_{1} p_{1} R_{1} U_{1}\right)\right] x y^{2}+p_{1}^{2} P_{1} U_{1} y^{3}$

Thus $\left\{\begin{aligned} x_{2}^{\prime}= & m_{2}+n_{2} x+p_{2} y+q_{2} x^{2}+r_{2} x y+s_{2} y^{2}+t_{2} x^{3}+ \\ & u_{2} x^{2} y+\hat{v}_{2} x y^{2}+w_{2} y^{3} \\ y_{2}^{\prime}= & M_{2}+N_{2} x+P_{2} y+Q_{2} x^{2}+R_{2} x y+S_{2} y^{2}+T_{2} x^{3} \\ + & U_{2} x^{2} y+V_{2} x y^{2}+W_{2} y^{3}\end{aligned}\right.$

where $m_{2}, n_{2}, p_{2}, \ldots . ., M_{2}, N_{2}, P_{2}, \ldots .$. etc. can be determined from above .

Proceeding in the same manner we can determine the expressions for any number of iterations of the map $f$.

\section{METHOD FOR THE COMPUTATION OF HOPF BIFURCATION POINTS}

We now develop here the eigenvalue theory of the Jacobian matrix of the vector field $f$. Suppose

$$
M=\left[\begin{array}{ll}
A & B \\
C & D
\end{array}\right]
$$

as the Jacobian matrix of $f$, then the eigenvalues $\eta$ of $M$ are given by

$\eta=\frac{1}{2}(A+D) \pm \frac{\sqrt{(A-D)^{2}+4 B C}}{2}=\phi \pm i \psi($ say $)$

(Here the patial derivatives $A, B, C$ and $D$ are taken so that that the eigenvalues $\eta$ come out as complex numbers)

If $\underline{e}=\left[\begin{array}{c}\mu \\ v\end{array}\right]$ is the eigenvector of the transpose of $M$ corresponding to the eigenvalue $\eta$, then

$$
\begin{aligned}
& {\left[\begin{array}{cc}
A-\eta & B \\
C & D-\eta
\end{array}\right]\left[\begin{array}{l}
\mu \\
v
\end{array}\right]=\left[\begin{array}{l}
0 \\
0
\end{array}\right] \Rightarrow \mu=C, v=\eta-A} \\
& =(\phi+i \psi)-A=(\phi-A)+i \psi
\end{aligned}
$$

Let us put $z=\mu x+v y$. This implies $\bar{z}=\bar{\mu} x+\bar{v} y$. Consequently

$x=\frac{\bar{v} z-v \bar{z}}{\bar{v} \mu-v \bar{\mu}}, y=\frac{\bar{\mu} z-\mu \bar{z}}{\bar{\mu} v-\mu \bar{\nu}} \Rightarrow x=\frac{\bar{v} z-v \bar{z}}{\sigma}, y=\frac{\bar{\mu} z-\mu \bar{z}}{\omega}$

where $\sigma=\bar{v} \mu-v \bar{\mu}=C(\bar{v}-v)=-2 i \psi C$ and

$\omega=\bar{\mu} \nu-\mu \bar{\nu}=-\sigma=2 i \psi C$

$$
z_{1}^{\prime}=\underline{e}^{T}=\mu \quad v\left[\begin{array}{l}
x^{\prime} \\
y^{\prime}
\end{array}\right] \text {, where } \underline{\mathrm{x}}=\left[\begin{array}{ll}
x^{\prime} & y^{\prime}
\end{array}\right]
$$

Now

$$
\begin{aligned}
= & \mu x^{\prime}+v y^{\prime} \\
= & \mu\left(q x^{2}+r x y+s y^{2}+t x^{3}+u x^{2} y+v x y^{2}+w y^{3}\right) \\
& +v\left(Q x^{2}+R x y+S y^{2}+T x^{3}+U x^{2} y+V x y^{2}+W y^{3}\right)
\end{aligned}
$$

(For simplicity, we omit the lower suffices from

$q, r, \ldots . ., Q, R, \ldots \ldots$ etc. Also to evaluate $H$ we do not need the coefficients of linear terms and hence the linear terms are omitted.)

$$
\begin{aligned}
& =(\mu q+v Q) x^{2}+(\mu r+v R) x y+(\mu s+v S) y^{2}+(\mu t+v T) x^{3} \\
& +(\mu u+v U) x^{2} y+(\mu \hat{v}+v V) x y^{2}+(\mu w+v W) y^{3} \\
& =Q^{\prime} x^{2}+R^{\prime} x y+S^{\prime} y^{2}+T^{\prime} x^{3}+U^{\prime} x^{2} y+V^{\prime} x y^{2}+W^{\prime} y^{3} \text { (say) }
\end{aligned}
$$

$=Q^{\prime}\left(\frac{\bar{v} z-v \bar{z}}{\sigma}\right)^{2}+R^{\prime}\left(\frac{\bar{v} z-v \bar{z}}{\sigma}\right)\left(\frac{\bar{\mu} z-\mu \bar{z}}{\omega}\right)+S^{\prime}\left(\frac{\bar{\mu} z-\mu \bar{z}}{\omega}\right)^{2}$ $+T^{\prime}\left(\frac{\bar{v} z-v \bar{z}}{\sigma}\right)^{3}+U^{\prime}\left(\frac{\bar{v} z-v \bar{z}}{\sigma}\right)^{2}\left(\frac{\bar{\mu} z-\mu \bar{z}}{\omega}\right)+V^{\prime}\left(\frac{\bar{v} z-v \bar{z}}{\sigma}\right)$ $\left(\frac{\bar{\mu} z-\mu \bar{z}}{\omega}\right)^{2}+W^{\prime}\left(\frac{\bar{\mu} z-\mu \bar{z}}{\omega}\right)^{3}=z^{2}\left[\frac{Q^{\prime} \bar{v}^{2}}{\sigma^{2}}+\frac{R^{\prime} \bar{v} \bar{\mu}}{\sigma \omega}+\frac{S^{\prime} \bar{\mu}^{2}}{\omega^{2}}\right]$ $+\mathrm{z} \overline{\mathrm{z}}\left[\frac{-2 Q^{\prime} \nu \bar{\nu}}{\sigma^{2}}+\frac{R^{\prime}(-\bar{v} \mu-v \bar{\mu})}{\sigma \omega}+\frac{-2 S^{\prime} \mu \bar{\mu}}{\omega^{2}}\right]+$ $=\bar{z}^{2}\left[\frac{Q^{\prime} v^{2}}{\sigma^{2}}+\frac{R^{\prime} v \mu}{\sigma \omega}+\frac{S^{\prime} \mu^{2}}{\omega^{2}}\right]+z^{2} z\left[\frac{T^{\prime}}{\sigma^{3}}\left(-3 \bar{v}^{2} v\right)+\right.$ $\frac{U^{\prime}}{\sigma^{2} \omega}\left(-\bar{v}^{2} \mu-2 v \bar{v} \bar{\mu}\right)+\frac{V^{\prime}}{\sigma \omega^{2}}\left(-\bar{\mu}^{2} v-2 \mu \bar{\mu} \bar{v}\right)+$ $\left.\frac{W^{\prime}}{\omega^{3}}\left(-3 \bar{\mu}^{2} \mu\right)\right]+\ldots \ldots$

$$
\begin{aligned}
Q^{\prime} \bar{v}^{2} & =(\mu q+v Q)(\phi-A-i \psi)^{2} \\
& =\left[\{\mu q+(\phi-A) Q\}\left\{(\phi-A)^{2}-\psi^{2}\right\}+2 \psi^{2} Q(\phi-A)\right]+ \\
& i\left[\{-2(\phi-A) \psi\}\{\mu q+(\phi-A) Q\}+\psi Q\left\{(\phi-A)^{2}-\psi^{2}\right\}\right] \\
& =e_{1}+i e_{2} \text { (say) }
\end{aligned}
$$




$$
\begin{aligned}
R^{\prime} \bar{\mu} \bar{v} & =\mu(\mu r+v R) \bar{v} \\
& =\left[\left\{\mu^{2} r+\mu R(\phi-A)\right\}(\phi-A)+\mu R \psi^{2}\right]+ \\
& i\left[-\psi\left\{\mu^{2} r+\mu R(\phi-A)\right\}+\mu R \psi(\phi-A)\right] \\
& =e_{3}+i e_{4}(\text { say }) \\
S^{\prime} \bar{\mu}^{2} & =(\mu s+v S) \mu^{2}=\{\mu s+S(\phi-A)+i S \psi\} \mu^{2} \\
& =\left\{\mu^{3} s+\mu^{2} S(\phi-A)\right\}+i \mu^{2} S \psi=e_{5}+i e_{6}, \text { (say) }
\end{aligned}
$$

Taking

$$
\begin{aligned}
A_{1} & =\left[\frac{Q^{\prime} \bar{v}^{2}}{\sigma^{2}}+\frac{R^{\prime} \bar{v} \bar{\mu}}{\sigma \omega}+\frac{S^{\prime} \bar{\mu}^{2}}{\omega^{2}}\right] \\
& =\frac{1}{-4 \psi^{2} \mu^{2}}\left[\left(e_{1}-e_{3}+e_{5}\right)+i\left(e_{21}-e_{4}+e_{6}\right)\right] \\
& =G_{0}+i H_{0},(\text { say })
\end{aligned}
$$

Again

$$
\begin{aligned}
& R^{\prime}(\bar{v} \mu+v \bar{\mu})=\mu R^{\prime}(\bar{v}+v)=-\mu(\mu r+v R)(\bar{v}+v) \\
& =2\left\{\mu^{2} r+\mu R(\phi-A)\right\}(\phi-A)+i\{2 \mu R \psi(\phi-A)\} \\
& =f_{3}+i f_{4}(\text { say }) \\
& 2 S^{\prime} \mu \bar{\mu}=2 \mu^{2}(\mu s+v S) \\
& =2 \mu^{2}\{\mu s+S(\phi-A)\}+i\left(2 \mu^{2} S \psi\right)=f_{5}+i f_{6},(\text { say }) \\
& B_{1}=\left[\frac{-2 Q^{\prime} v_{\bar{v}}}{\sigma^{2}}-\frac{R^{\prime}(\bar{v} \mu+v \bar{\mu})}{\sigma \omega}-\frac{2 S^{\prime} \mu \bar{\mu}}{\omega^{2}}\right] \\
& =\frac{1}{4 \mu^{2} \psi^{2}}\left[\left(f_{1}-f_{3}+f_{5}\right)+i\left(f_{2}-f_{4}+f_{6}\right)\right] \\
& =I_{0}+i J_{0},(\text { say })
\end{aligned}
$$

Also

$$
\begin{aligned}
Q^{\prime} v^{2} & =\left[\{\mu q+(\phi-A) Q\}\left\{(\phi-A)^{2}-\psi^{2}\right\}-2 \psi^{2} Q(\phi-A)\right]+ \\
& =i\left[\{\mu q+(\phi-A) Q\}\{2 \psi(\phi-A)\}+\psi Q\left\{(\phi-A)^{2}-\psi^{2}\right\}\right] \\
& =g_{1}+i g_{2}(\text { say })
\end{aligned}
$$

$$
\begin{aligned}
R^{\prime} v \mu= & \mu\left[\left\{(\mu r+R(\phi-A)\}(\phi-A)-\psi^{2} R\right]+\right. \\
& i \mu[\psi\{\mu r+R(\phi-A)\}+\psi R(\phi-A)] \\
= & g_{3}+i g_{4} \text { (say) }
\end{aligned}
$$

$$
\begin{aligned}
S^{\prime} \mu^{2} & =\left\{\mu^{3} s+\mu^{2} S(\phi-A)\right\}+i \mu^{2} \psi S \\
& =g_{5}+i g_{6}(\text { say })
\end{aligned}
$$

$$
\begin{aligned}
& -3 T \bar{v}^{2} v=-3(\mu t+v T) \bar{v}^{2} v \\
& =-3[\mu t+\{T(\phi-A)+i \psi T\}]\left(l_{1}+i l_{2}\right) \\
& =-3\left[\{\mu t+T(\phi-A)\} l_{1}-\psi T l_{2}\right]+i(-3)\left[\psi T l_{1}+\right. \\
& \left.l_{2}\{\mu t+T(\phi-A)\}\right]=j_{1}+i j_{2} \text { (say), } \\
& \text { where } \bar{v}^{2} v=\left[\left\{(\phi-A)^{2}-\psi^{2}\right\}(\phi-A)+\right. \\
& \left.2 \psi^{2}(\phi-A)\right]+i\left[\psi\left\{(\phi-A)^{2}-\psi^{2}\right\}-\right. \\
& \left.2 \psi(\phi-A)^{2}\right]=l_{1}+i l_{2} \text { (say) } \\
& -U^{\prime}\left(\bar{v}^{2}+2 v \bar{v}\right) \mu \\
& =-\mu\left[\{\mu u+U(\phi-A)\}\left\{3(\phi-A)^{2}+\psi^{2}\right\}\right. \\
& \left.+2 \psi^{2} U(\phi-A)\right]+i(-\mu)[-2\{\mu u+U(\phi-A)\} \\
& \left.\psi(\phi-A)+\psi U\left\{3(\phi-A)^{2}+\psi^{2}\right\}\right] \\
& =j_{3}+i j_{4} \text { (say), } \\
& -V^{\prime}\left(\bar{\mu}^{2} v+2 \mu \bar{\mu} \bar{v}\right) \\
& =\left(-\mu^{2}\right)\left[\{\mu \hat{v}+V(\phi-A)\} 3(\phi-A)+\psi^{2} V\right] \\
& +i\left(-\mu^{2}\right)[3 \psi V(\phi-A)-\psi\{\mu \hat{v}+V(\phi-A)\}] \\
& =j_{5}+i j_{6} \text { (say), } \\
& -3 W^{\prime} \mu \bar{\mu}^{2}=-3 \mu^{3}\{\mu w+W(\phi-A)\}+i\left(-3 \mu^{3} \psi W\right) \\
& =j_{7}+i j_{8}(\text { say }) \text {, } \\
& M_{1}=\left[\frac{T^{\prime}}{\sigma^{3}}\left(-3 \bar{v}^{2} v\right)-\frac{U^{\prime}}{\sigma^{2} \omega}\left(\bar{v}^{2} \mu+2 v \overline{v \mu}\right)-\frac{V^{\prime}}{\sigma \omega^{2}}\left(\bar{\mu}^{2} v\right.\right. \\
& \left.+2 \mu \bar{\mu} \bar{\nu})+\frac{W^{\prime}}{\omega^{3}}\left(-3 \bar{\mu}^{2} \mu\right)\right]=\frac{1}{8 \psi^{3} \mu^{3}}\left[\left(j_{1}-j_{4}-j_{6}+j_{7}\right)+\right. \\
& \left.i\left(j_{2}+j_{3}+j_{5}+j_{8}\right)\right]=R_{0}+i S_{0},(\text { say }) \\
& \eta=\phi+i \psi \Rightarrow \eta^{2}=\phi^{2}-\psi^{2}+i 2 \phi \psi \\
& \Rightarrow \eta^{3}=\left(\phi^{3}-3 \phi \psi^{2}\right)+i\left(3 \psi \phi^{2}-\psi^{3}\right) \\
& =K_{1}+i K_{2}(\text { say }) \eta^{3}-\eta^{2}=\left(\phi^{3}-\phi^{2}\right. \\
& \left.-3 \phi \psi^{2}+\psi^{2}\right)+i\left(3 \psi \phi^{2}-2 \phi \psi-\psi^{3}\right) \\
& =A_{1}^{\prime}+i A_{2}^{\prime}(\text { say })(1-2 \eta) A_{1} B_{1}=(1-2 \phi \\
& -2 i \psi)\left(G_{0}+i H_{0}\right)\left(I_{0}+i J_{0}\right)=[\{(1-2 \phi) \\
& \left.\left.G_{0}+2 \psi H_{0}\right\} I_{0}-\left\{(1-2 \phi) H_{0}-2 \psi G_{0}\right\} J_{0}\right]+ \\
& i\left[\left\{(1-2 \phi) G_{0}+2 \psi H_{0}\right\} J_{0}+\right. \\
& \left.\left\{(1-2 \phi) H_{0}-2 \psi G_{0}\right\} I_{0}\right]=A_{3}^{\prime}+i A_{4}^{\prime} \text { (say) }
\end{aligned}
$$

After calculating above somewhat complicated expressions,

$$
\begin{aligned}
& H=\frac{(1-2 \eta) A_{1} B_{1}}{\eta^{3}-\eta^{2}}+\frac{B_{1} \bar{B}_{1}}{\eta-1}+\frac{2 C_{1} \bar{C}_{1}}{\eta^{3}-1}+\eta^{-1} M_{1} \\
& \frac{(1-2 \eta) A_{1} B_{1}}{\eta^{3}-\eta^{2}}=\frac{A_{3}^{\prime}+i A_{4}^{1}}{A_{1}^{\prime}+i A_{2}^{\prime}}=\frac{\left(A_{3}^{\prime}+i A_{4}^{1}\right)\left(A_{1}^{\prime}-i A_{2}^{\prime}\right)}{A_{1}^{\prime 2}+A_{2}^{\prime 2}} \\
& \Rightarrow \operatorname{Re}\left(\frac{(1-2 \eta) A_{1} B_{1}}{\eta^{3}-\eta^{2}}\right)=X_{1}^{00}(\text { say })
\end{aligned}
$$$$
\text { we have the stability index } \boldsymbol{H} \text { as }
$$ 


$$
\begin{aligned}
& \quad \frac{B_{1} \bar{B}_{1}}{\eta-1}=\frac{I_{0}^{2}+J_{0}^{2}}{(\phi+i \psi)-1}=\frac{\left(I_{0}^{2}+J_{0}^{2}\right)(\phi-1-i \psi)}{(\phi-1)^{2}+\psi^{2}} \\
& \Rightarrow \operatorname{Re}\left(\frac{B_{1} \bar{B}_{1}}{\eta-1}\right)=X_{2}^{00}(\text { say }) \\
& \frac{2 C_{1} \bar{C}_{1}}{\eta^{3}-1}=\frac{2\left(P_{0}^{2}+Q_{0}^{2}\right)}{\left(K_{1}-1\right)+i K_{2}}=\frac{2\left(P_{0}^{2}+Q_{0}^{2}\right)\left\{\left(K_{1}-1\right)-i K_{2}\right\}}{\left(K_{1}-1\right)^{2}+K_{2}^{2}} \\
& \Rightarrow \operatorname{Re}\left(\frac{2 C_{1} \bar{C}_{1}}{\eta^{3}-1}\right)=X_{3}^{00}(\text { say }) \\
& \eta^{-1} M_{1}=\frac{M_{1}}{C}=\frac{R_{0}+i S}{\phi+i \psi} \\
& \Rightarrow \operatorname{Re} \eta^{-1} M_{1}=\frac{\phi R_{0}+\psi S_{0}}{\phi^{2}+\psi^{2}} \\
& =X_{4}^{00}(\text { say })
\end{aligned}
$$

$\begin{array}{llll}-0.720000 & -0.360000 & 0.921737 & -2.580188 \\ -0.740000 & -0.370000 & 0.936429 & -1.961760 \\ -0.760000 & -0.380000 & 0.950999 & -1.362047 \\ -0.780000 & -0.390000 & 0.965453 & -0.819575 \\ -0.800000 & -0.400000 & 0.979796 & -0.364137 \\ -0.820000 & -0.410000 & 0.994032 & -0.010263\end{array}$

Under this situation, we have

$\operatorname{Re} H=X_{1}^{00}+X_{2}^{00}+X_{3}^{00}+X_{4}^{00}$

Analogous theory can be developed for any number of

\begin{tabular}{|c|c|c|c|}
\hline$P V$ & $R E V$ & IEV & $H$ \\
\hline-0.840000 & 35.547664 & 35.411240 & 0.872926 \\
\hline-0.845000 & 35.645886 & 35.509420 & 0.873715 \\
\hline-0.850000 & 35.744326 & 35.607817 & 0.874490 \\
\hline-0.855000 & 35.842983 & 35.706433 & 0.875251 \\
\hline-0.860000 & 35.941858 & 35.805266 & 0.875998 \\
\hline-0.865000 & 36.040951 & 35.904318 & 0.876731 \\
\hline-0.870000 & 36.140262 & 36.003589 & 0.877451 \\
\hline-0.875000 & 36.239791 & 36.103077 & 0.878157 \\
\hline-0.880000 & 36.339537 & 36.202784 & 0.878850 \\
\hline-0.885000 & 36.439502 & 36.302710 & 0.879529 \\
\hline-0.890000 & 36.539684 & 36.402853 & 0.880195 \\
\hline-0.895000 & 36.640085 & 36.503216 & 0.880848 \\
\hline-0.900000 & 36.740704 & 36.603797 & 0.881488 \\
\hline-0.905000 & 36.841541 & 36.704597 & 0.882115 \\
\hline-0.910000 & 36.942597 & 36.805615 & 0.882728 \\
\hline-0.915000 & 37.043871 & 36.906853 & 0.883330 \\
\hline-0.920000 & 37.145363 & 37.008309 & 0.883918 \\
\hline-0.925000 & 37.247074 & 37.109984 & 0.884494 \\
\hline
\end{tabular}
iterations of the map $f$. To evaluate the values of $H$ we compose a computer program and obtain the values of $H$ for the maps $f$ and $f^{2}$ as tabulated below in tables 1.1 and 1.2.

\section{Table 1.1 (For the map $f$ )}

$\begin{array}{cccc}P V & R E V & I E V & H \\ -0.420000 & -0.210000 & 0.681249 & -8.711063 \\ -0.440000 & -0.220000 & 0.698856 & -8.197007 \\ -0.460000 & -0.230000 & 0.716170 & -7.744707 \\ -0.480000 & -0.240000 & 0.733212 & -7.342201 \\ -0.500000 & -0.250000 & 0.750000 & -6.978884 \\ -0.520000 & -0.260000 & 0.766551 & -6.644871 \\ -0.540000 & -0.270000 & 0.782879 & -6.330438 \\ -0.560000 & -0.280000 & 0.798999 & -6.025507 \\ -0.580000 & -0.290000 & 0.814923 & -5.719211 \\ -0.600000 & -0.300000 & 0.830662 & -5.399610 \\ -0.620000 & -0.310000 & 0.846227 & -5.053740 \\ -0.640000 & -0.320000 & 0.861626 & -4.668285 \\ -0.660000 & -0.330000 & 0.876869 & -4.231255 \\ -0.680000 & -0.340000 & 0.891964 & -3.734987 \\ -0.700000 & -0.350000 & 0.906918 & -3.180244\end{array}$

Table 1.2 (For the map $f^{2}$ )

In both of these tables $P V, R E V$ and $I E V$ mean parameter value, $x$-coordinate of fixed point, $y$-coordinate of fixed point, real part of eigenvales and imaginart part of eigenvales respectively. 


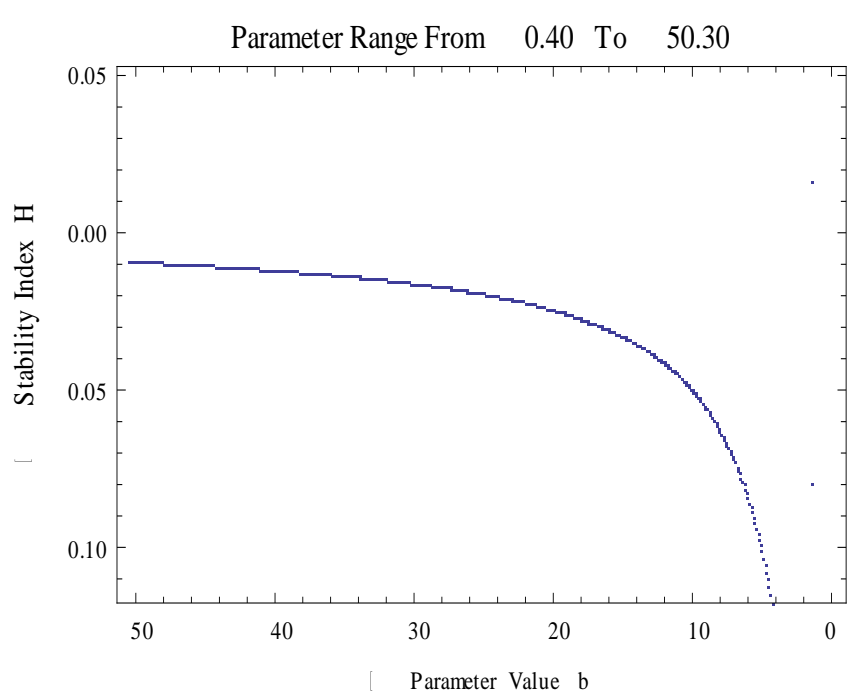

Figure 1.1 five hundred points are plotted for the map $f$

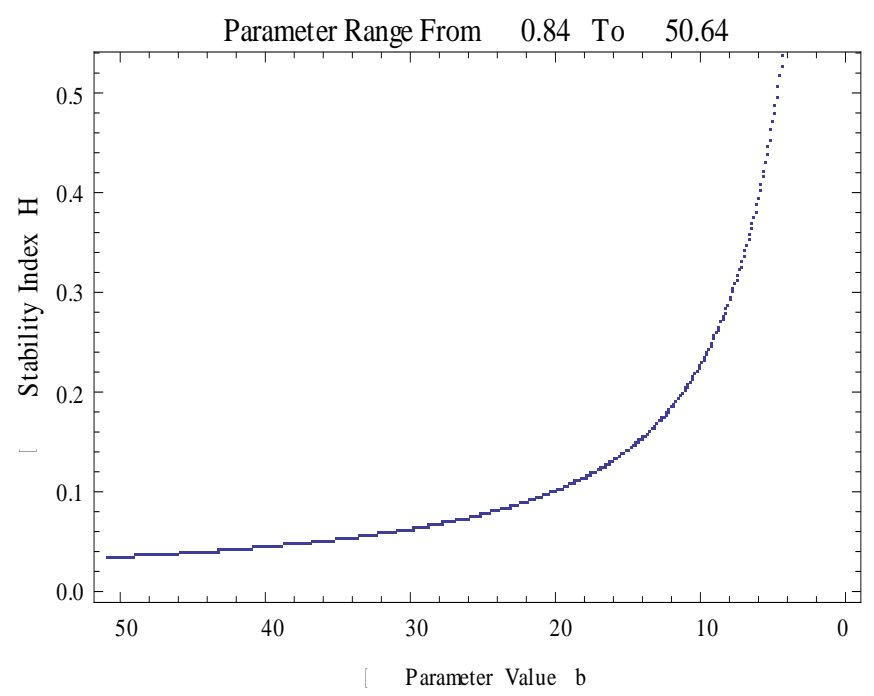

Figure 1.2 five hundred points are plotted for the map $f^{2}$.

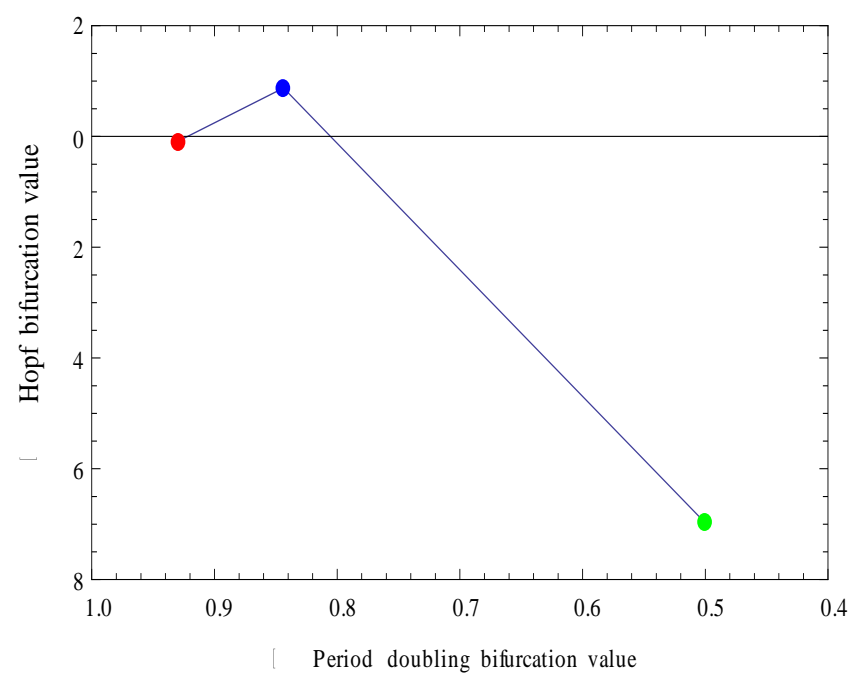

Figure 1.3 Period-doubling and Hopf bifurcation curve
From the above tables and figures we have found that while the map $f$ undergoes supercritical Hopf bifurcation and $f^{2}$ undergo subcritical Hopf bifurcation. Furthermore, the graph in the Figure 1.3 shows that there is a close link between Hopf bifurcation and Period-doubling bifurcation.

\section{Period-doubling and Hopf bifurcation values:}

$\begin{array}{lll}\text { Map } & \begin{array}{l}\text { Period-doubling } \\ \text { bifurcation value }\end{array} & \text { Hopf bifurcation value } \\ f & -0.5 & -6.978883861237 \ldots \\ f^{2} & -0.843730536895 \ldots & 0.873515810209 \ldots \\ f^{4} & -0.9295533 \ldots & -0.000000936344 \ldots\end{array}$

\section{Period-Doubling Tree}

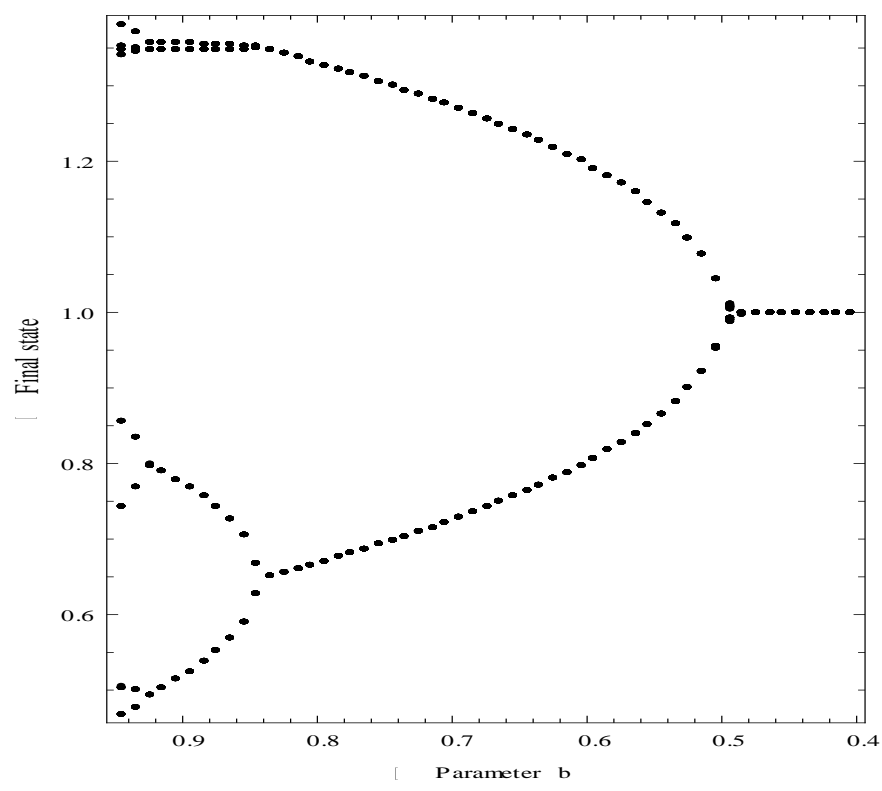

Figure 1.4 The Period-Doubling Tree for the parameter range $-0.945 \leq \mathrm{b} \leq-0.4$

\section{CONCLUSIONS}

The theory described in this paper can be used to determine the Hopf bifurcation values of any nonlinear maps and the behavior of Hopf bifurcation changes from supercritical to subcritical or vice-versa at the period-doubling points.

\section{REFERENCES}

[1] Das, N. and Dutta, T. K., Determination of supercritical and subcritical Hopf bifurcation on a two-dimensional chaotic model, International Journal of Advanced Scientific Research and Technology, Issue2, Vol. 1, February, 2012

[2] Davie, A. M. and Dutta, T K, Period-doubling in twoparameter families, Physica D, 64 (1993), 345- 354 
[3] Dutta T. K. and Das, N., Period Doubling Route to Chaos in a Two-Dimensional Discrete Map, Global Journal of Dynamical Systems and Applications, Vol.1, No. 1, pp. 6172,2001

[4] Hilborn, Robert C., Chaos and Nonlinear Dynamics, Oxford University Press, 1994

[5] Hopf, E., Abzweigung einer periodischen Losung von einer stationaren Losung eines Differential systems, Ber. Verh. Sachs. Akad. Wiss. Leipsig Math.-Nat. 94(1942), 3-22, Translation to English with commentary by L. Howard and N. Kopell, in[81; 163-205]

[6] Marsden, J. E. and McCracken, M., The Hopf Bifurcation and Its Applications, Springer-Verlag, New York, 1976

[7] Moiola, J. L. and Chen, G., Hopf Bifurcation Analysis: a frequency domain approach, World Scientific, 1996

[8] Roose, D. and Hlavacek, V., A Direct Method for the computation of Hopf bifurcation points, SIAM J. APPL. MATH., Vol. 45, No. 6, December 1985

[9] Sandri Marco, Numerical calculation of Lyapunov Exponents, University of Verona, Italy

[10] Steeb, Willi-Hans in collaboration with Hardy, Y. and Stoop, R., The Nonlinear Workbook: $\quad\left(4^{\text {th }}\right.$ Edition), World Scientific, 2008 